\title{
Kangfuxinye Enema Combined with Mesalamine for Ulcerative Colitis: A Systematic Review and GRADE Approach
}

\author{
Peng-wei Ren,, ${ }^{1}$ Wen-jie Yang, ${ }^{1}$ Dan-dan Wang, ${ }^{1}$ Jing-yan Shan, \\ De-ying Kang, ${ }^{1}$ Qi Hong, ${ }^{1}$ Shu Wen, ${ }^{3}$ and Ru-wen Zhang ${ }^{4}$ \\ ${ }^{1}$ Department of Evidence-Based Medicine and Clinical Epidemiology, West China Hospital, Sichuan University, \\ Chengdu 610041, China \\ ${ }^{2}$ West China School of Public Health, No. 4 West China Teaching Hospital, Sichuan University, Chengdu 610041, China \\ ${ }^{3}$ West China School of Medicine and West China Hospital, Sichuan University, Chengdu 610041, China \\ ${ }^{4}$ Department of Integrated Traditional Chinese and Western Medicine, West China Hospital, Sichuan University, \\ Chengdu 610041, China \\ Correspondence should be addressed to De-ying Kang; deyingkang@126.com
}

Received 16 December 2016; Accepted 21 June 2017; Published 7 August 2017

Academic Editor: Steve Harakeh

Copyright (C) 2017 Peng-wei Ren et al. This is an open access article distributed under the Creative Commons Attribution License, which permits unrestricted use, distribution, and reproduction in any medium, provided the original work is properly cited.

Objectives. To critically appraise the efficacy and safety of Kangfuxinye enema combined with mesalamine for the ulcerative colitis (UC) patients and in addition to grade the quality of evidence by using the GRADE (grading of recommendations, assessment, development, and evaluation) approach. Methods. A literature search was performed in the Cochrane Library, MEDLINE, EMBASE, CBM, CNKI, VIP, and WanFang Databases. The search restrictions were patients with UC and RCTs. Studies including other treatments except Kangfuxinye with mesalamine were excluded. Results. Nineteen studies met the inclusion criteria. We found significant benefits of Kangfuxinye combined with mesalamine against mesalamine alone in improving response rate as well as reducing the recurrence rate and inflammation rate; meanwhile, the increase of the adverse events rate was not observed. Furthermore, the symptoms remission rate and the cure time were insignificant statistically. Additionally, GRADE results indicated that the quality of evidence regarding the above 6 outcomes was rated from very low to moderate quality. Conclusions. Although Kangfuxinye enema seems effective and safe for treating UC patients in this systematic review, Kangfuxinye enema combined with mesalamine was weakly recommended due to very low to moderate quality of available evidence by the GRADE approach.

\section{Introduction}

Ulcerative colitis (UC) is one of the 2 major types of inflammatory bowel disease (IBD), along with Crohn disease but 3 times more common compared to it $[1,2]$. The incidence of UC is 1.2-20.3 cases per 100,000 per year, and the developed countries, such as Northern Europe and North America, have the highest incidence of the disease $[1,3]$. In Asia and the Middle East, the incidence is about 6.3 per 100,000 personyears. Universally, UC occurs mainly between the second and fourth decades of life [4]. In combination with the change of environment and other unknown reasons, UC has become a global emergence disease with increasing incidence and prevalence worldwide [5]. Typical symptoms of UC include abdominal pain, tenesmus, bloody diarrhea, passage of pus, mucus, or both, urgency, weight loss, and fever [6], which causes a miserable influence on the quality of life of the UC patients. Moreover, UC affects individuals in their most formidable and productive years of life, resulting in heavy burden on the patients' life, health care system, and society [7]. In addition, high relapse rates and protracted courses of disease also lead to the increasing risk of colorectal cancer $[8,9]$. Therefore, UC often requires life-long maintenance therapy for relieving symptoms and/or to attenuate the inflammation while there is lack of curative treatment.

Mesalamine (USAN), also known as mesalazine (INN, BAN) or 5-aminosalicylic acid (5-ASA), is most commonly used as a first-line therapy for mild to moderate UC [10]. 
However, the majority of patients with UC exhibited low adherence and persistence to mesalamine, which has been an important barrier for successful management [11]. Indeed, the major consequences of nonadherence to 5-ASA for UC patients had a fivefold higher risk of relapse, an increased risk of colorectal cancer, and a reduced quality of life [8]. Once the first-line therapy fails, patients would turn to alternative medicine such as steroids [12], azathioprine [13], and the antitumour necrosis factor alpha (TNFa) agent infliximab [14]. Nevertheless, those alternative therapies always accompany increased risks of infection and malignancy.

At present, complementary and alternative medicine (CAM) is increasingly applied for treatment of IBD due to its potential efficacy $[15,16]$, and it accounts for about $21 \%$ of inflammatory bowel disease patients now [17]. Of those, Kangfuxinye, a pure Chinese herbal medicine extracted from the Periplaneta americana, has been widely used for treating ulcerative and inflammatory diseases $[18,19]$ due to its sound effects on anti-inflammatory and recovery of gastrointestinal mucosal, and animal studies have also suggested that the therapeutic effect of Kangfuxinye may be due, at least in part, to its stimulatory effect on nonspecific cellular defense mechanisms [20], making it one of the most addressed therapies for UC, especially in Chinese UC patients. Although previous studies had shown sound effects of Kangfuxinye for treating UC patients, the quality of the studies has become a common concern, thus further researches are needed before making recommendations for clinical practice. One previous systematic review (SR) [21] indicated Kangfuxinye having short-term benefits regarding the overall response and inflammation reduction, but its safety and long-term effect still remain unclear. In addition, the quality of evidence needs to be appraised and validated critically.

Therefore, the aims of this study were to systematically review the efficacy and safety of Kangfuxinye enema in combination with mesalamine according to the Cochrane Collaboration's guidance for SR and then to grade quality of the evidence and make recommendations for practice by using The Grading of Recommendations, Assessment, Development and Evaluation (GRADE) approach [22] which is always used as an instrument for grading quality of evidence within systematic reviews and guidelines and for making evidence-based recommendations during guidelines development [23].

\section{Methods}

This study was conducted using the Cochrane Collaboration's approach [43] and this systematic review is consistent with the PRISMA (the Preferred Reporting Items for Systematic Reviews and Meta-Analyses) checklist [44]. In addition, the GRADE approach [22] was also taken to grade the quality of evidence and make recommendation regarding the use of Kangfuxinye enema in the UC. Five methodological factors (risk of bias, inconsistency, indirectness, imprecision, and publication bias) were judged to downgrade or upgrade the quality of evidence [45]. Ethical approval and patient informed consent were waived because all data were extracted from previous studies.
TABLE 1: Rating scale for outcome ranking according to clinical importance.

\begin{tabular}{lc}
\hline Importance & Measure \\
\hline Critical $^{*}$ & Recurrence rate \\
& Response rate \\
Important $^{\dagger}$ & Inflammation reduction rate \\
& Symptom remission rate \\
& Adverse effects rate \\
Not important $^{\ddagger}$ & Time of cure \\
\hline
\end{tabular}

${ }^{*}$ Critical for making a decision and included in the evidence profile. ${ }^{\dagger}$ Important for making a decision and included in the evidence profile. ${ }^{\ddagger}$ Not important for making a decision and not included in the evidence profile.

\subsection{Criteria for Considering Studies for This Review}

2.1.1. Type of Studies. Only RCTs, which were published or unpublished in English or Chinese, were identified for this review. Observational studies, quasi-randomized controlled trials (Q-RCTs), controlled clinical trials (CCTs) were excluded.

2.1.2. Types of Participants. Participants (male/female) diagnosed with UC and who met the indications for using Kangfuxinye as enema were included in this study.

2.1.3. Types of Interventions. Kangfuxinye enema combined with mesalamine served as the intervention and taking mesalamine alone served as the control. Any mode of the mesalamine was eligible for this review.

2.1.4. Types of Outcome Measures. We consulted with 5 clinicians specialized in UC from West China hospital, to identify possible outcomes relating to the UC's efficacy and safety as well as to rate clinical importance of each outcome with assigning a value of 1 (lowest importance) to 9 (highest importance). The results were then used to generate a mean score with standard deviation (SD) for each outcome. The importance of each outcome was classified according to the mean score. Three outcome categories were identified regarding the clinical importance: critical (mean score of 7-9), important but not critical (mean score of 4-6), and limited importance (mean score of 1-3) [22]. Critical and important outcomes in Table 1 were used to make recommendations (Table 1).

\subsection{Search Strategies}

2.2.1. Electronic Searches. The following databases were searched from the inception through March 31, 2016: Cochrane Central Register of Controlled Trials (CENTRAL, Ovid), MEDLINE (PubMed), EMBASE (Ovid), Chinese Biomedicine Database (CBM), China National Knowledge Infrastructure (CNKI), VIP Information Database (VIP), and WanFang Database. The search terms used were "Kangfuxinye"; "Mesalamine"; and "ulcerative colitis" in Chinese or English. 
2.2.2. Search Other Sources. We also screened reference list of all obtained papers. Additionally, conference proceedings and dissertation abstracts were retrieved to identify unpublished studies.

2.3. Selection of Studies. Retrieved records including titles and abstracts were screened independently by 2 reviewers (P-W R and W-J Y) using EndNote 5.0 software after removal of duplications. The studies were included if they were Kangfuxinye enema combined with mesalamine against mesalamine alone. Observational studies, quasi-randomized controlled trials (Q-RCTs), controlled clinical trials (CCTs), and trials with paired interventions besides Kangfuxinye and mesalamine were excluded. Dissertations and abstracts were included when they contained sufficient details. All of the eligible studies were downloaded. Discrepancies were resolved via discussion or in consultation with the third reviewer (D-Y K).

2.4. Data Extraction and Management. All studies were reviewed by two reviewers (P-W R, W-J Y), who extracted data from the studies with the predeveloped forms including items such as the following: first author, publication year, sample size in each group, characteristics of participants (including age, sex, and degree of UC), diagnosis criteria of UC, details of Kangfuxinye enema and mesalamine, measured outcomes, follow-up (where available), and the number and reasons of missing participants.

Mean score changes from baseline to a particular endpoint were also abstracted. If unavailable, we extracted mean scores of baseline and endpoint as well as the SDs [43, 46]. Consensus was obtained by discussion or by consulting the third reviewer (D-Y K).

2.5. Assessment of Risk of Bias in Included Studies. Risk of bias for each eligible study was assessed by 2 reviewers (PW R and W-J Y) using the Cochrane Collaboration's Risk of Bias Tool in 6 domains: random sequence generation, incomplete outcome measures, blinding of participants and personnel, and outcome assessors, and allocation concealment, and selective outcome reporting [43]. Disagreements were resolved by discussion between the two reviewers (PW R, W-J Y), or with the arbitration of a third reviewer (DY K) being sought if necessary. There was no disagreement between the two reviewers on the risk of bias.

2.6. Data Synthesis and Statistical Analysis. A meta-analysis was performed by using the Review Manager (Version 5.3 for Windows; Cochrane Collaboration, Oxford, UK) if needed. For dichotomous data, pooled effect estimate was calculated using risk ratio (RR) with its 95\% confidence interval (CI). For continuous data, overall treatment effect size was calculated using mean difference (MD) with its $95 \%$ CI when the same rating scale was used, or using standardized mean difference (SMD) if rating scales were different. A 2sided $P \leq 0.05$ was considered as the threshold for statistical significance. Heterogeneity across study results was assessed using Cochrane's $Q$ statistic with $P$ value. $I^{2}$ statistic was used to quantify the degree of heterogeneity. If $P<0.1$ or $I^{2}>50 \%$, this indicates significant heterogeneity was present [43], and a random-effects model was applied to pool overall effect estimate; otherwise, a fixed-effects model was used. Subgroup analyses were carried out where available to investigate potential influence of clinical characteristics of participants or methodological quality on treatment effect size. Sensitivity analyses were performed where available to explore possible heterogeneity and its impact on the robustness of study results. If the number of included studies was sufficient $(n>10)$, a funnel plot or Egger's regression test was generated to detect potential publication bias $[47,48]$.

2.7. The GRADE Approach. Quality of evidence for each specific outcome among the included studies was evaluated by using the GRADE approach. Two authors (P-W R and W-J Y) received training on how to use GRADEro [49] in the 23nd Cochrane Colloquium (Vienna, Austria, from October 3 to 7, 2015), and separately assessed the quality in the estimate of each outcome. The evidence quality across each outcome was upgraded or downgraded determined by 5 primary domains (risk of bias, inconsistency, indirectness, imprecision, and publication bias) and was eventually categorized into 4 levels (high, moderate, low, and very low) [23].

\section{Results}

Our searches identified 202 potentially relevant studies, of which 193 references were all from electronic databases, 9 references from relevant reference lists, and no references were obtained from conference proceedings or dissertation abstracts. Finally, 19 studies [24-42] from electronic databases met our inclusion criteria. Further details were shown in Figure 1.

3.1. Characteristics of Included Studies. The characteristics of all included RCTs [24-42] were listed in Table 2. All RCTs were conducted in China and were published in Chinese. Males approximately account for half of the enrolled patients in each study. No dropouts were observed in these studies.

3.2. Assessment of Risk of Bias in Included Studies. The risk of bias of all included RCTs was assessed by using Cochrane Collaboration risk of bias tool. Because of inadequate reporting of randomization sequence generation and allocation concealment, all of the two items were judged as "unclear" which means that the potential risk of selection bias may exist. Of those, only two RCTs [24-42] used random number table to produce random sequence, whereas other trials just reported "randomly assigned" but failed to report on how sequence is produced. Details of allocation being concealed were unclear in all studies. Meanwhile, whether other important risks of bias existed could not be assessed due to paucity of data among the included trials. Overall, the included RCTs had moderate or high risks of bias in terms of 6 domains (Table 3 ). 


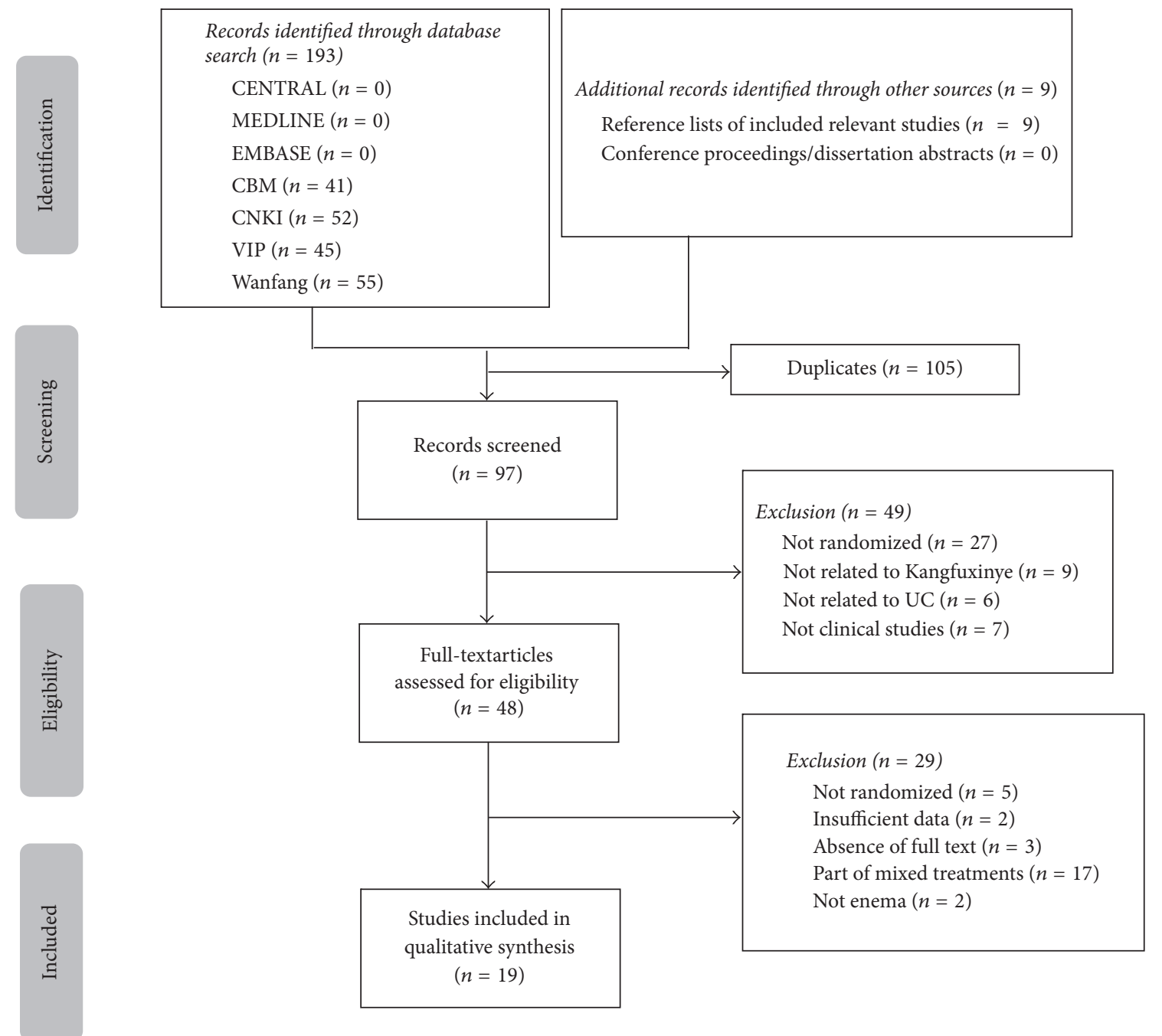

FIGURE 1: Flow diagram of the selection process. CENTRAL = Cochrane Central Register of Controlled Trials; CBM = Chinese Biomedicine Database; CNKI = China National Knowledge Infrastructure; VIP: Chinese Scientific Journals Database.

\subsection{Critical Outcomes}

3.3.1. Recurrence Rate. Five RCTs [24, 26, 29, 39, 41] including 360 patients reported recurrence rate. Recurrence was monitored after 3 12 months of follow-up among these trials. Compared with mesalamine, the meta-analysis indicated that Kangfuxinye combined with mesalamine enema reduced recurrence significantly $(\mathrm{RR}=0.33,95 \% \mathrm{CI}: 0.20-0.53, P<$ $0.001)$ without heterogeneity $\left(I^{2}=0 \%, P=0.99\right)$ (Figure 2$)$. A GRADE analysis indicated that the quality of evidence supporting this outcome was moderate due to risk of bias (Table 4).

3.3.2. Response Rate. 16 RCTs [24-31, 33, 36-42] including 1236 patients reported response rate. The outcome measure was based on both physician's assessment and the results of endoscopy typically divided into four categories, including (1) recovery, (2) significant improvement, (3) mild improvement, and (4) no change. The meta-analysis suggested favourable effects of Kangfuxinye combined with mesalamine against mesalamine $(\mathrm{RR}=1.19,95 \% \mathrm{CI}=1.14$ to $1.25, P<0.0001$; heterogeneity: $I^{2}=0 \%, P=0.89$ ) (Figure 3). A GRADE approach indicated that the quality of evidence supporting this outcome was low due to serious risk of bias (Table 4).

\subsection{Important Outcomes}

3.4.1. Inflammation Reduction Rate. Of those included 5 trials [25, 30, 33-35] providing examination of the inflammation reduction by endoscopy and endoscopy grading or scoring systems for inflammatory bowel diseases (IBD), a significant difference on the inflammation reduction rate was observed between two groups (fixed-effects model, RR $=1.30,95 \%$ CI: $1.16-1.46, P<0.001)$ without heterogeneity $\left(I^{2}=0 \%\right.$, $P=0.44$ ) (Figure 4). A GRADE approach indicated that the quality of evidence supporting this outcome was low due to serious risk of bias (Table 4).

3.4.2. Symptom Remission Rate. Four studies [30, 32, 34, 35] including 269 patients reported symptom remission rate. The 


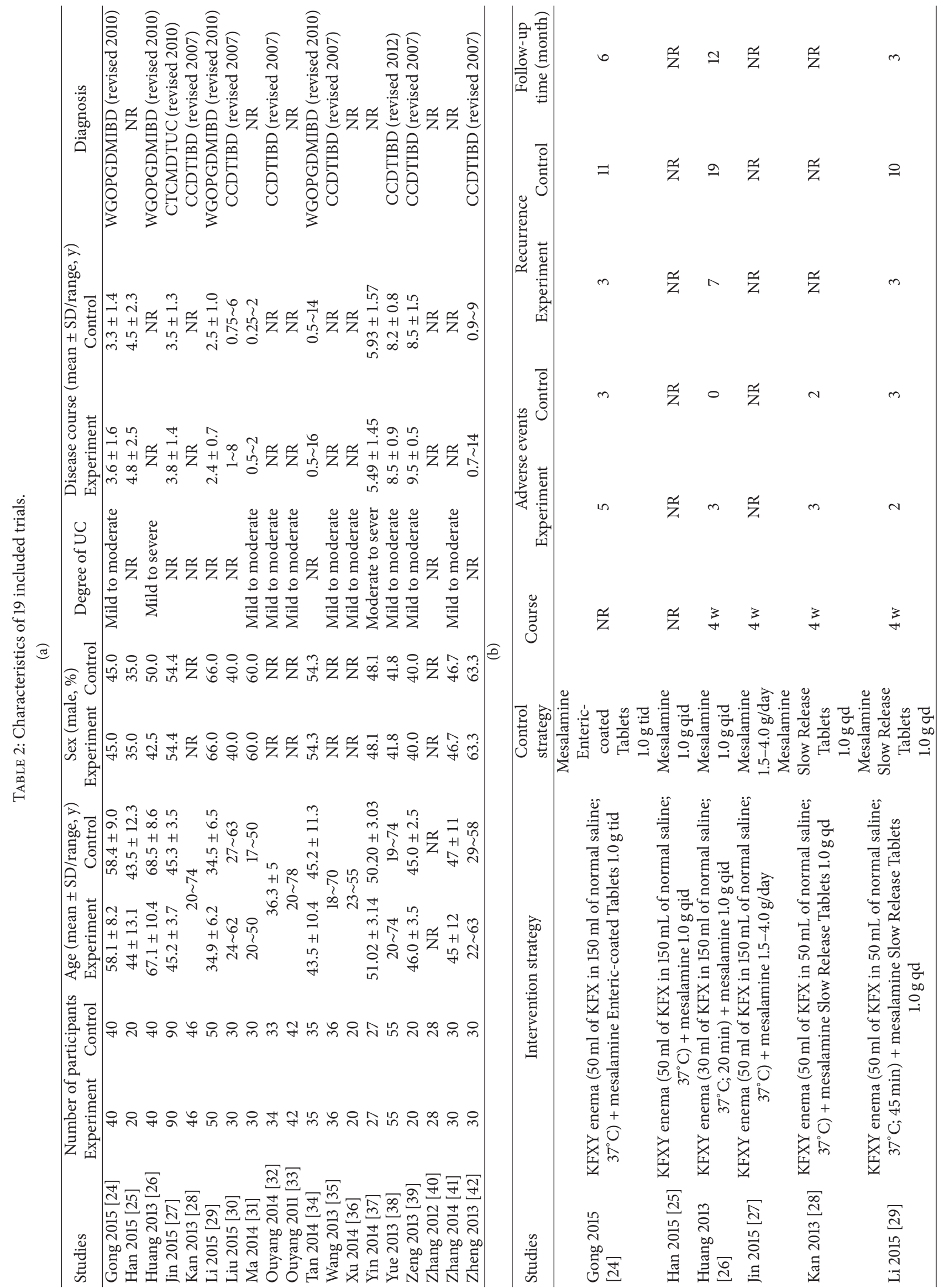




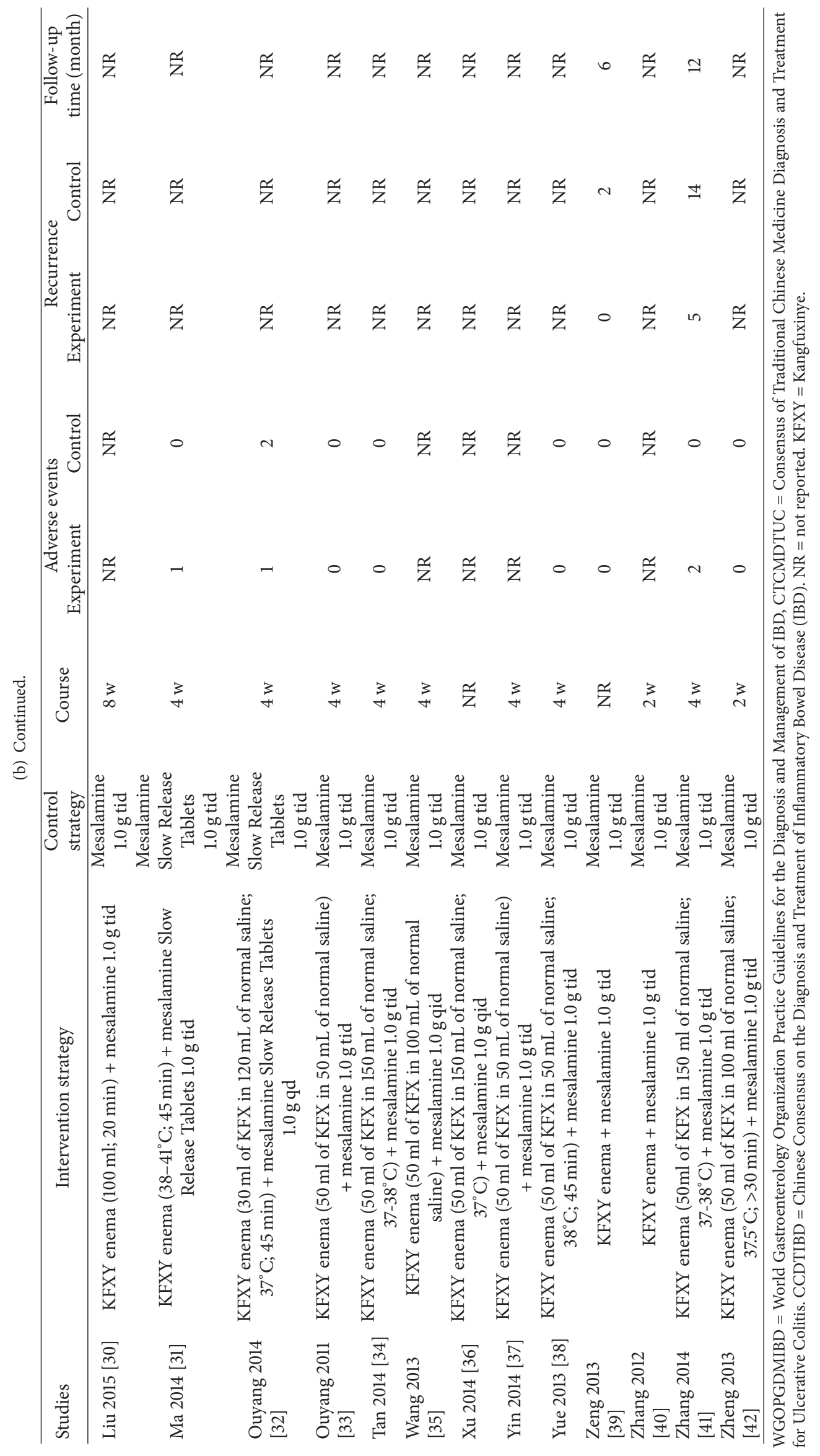




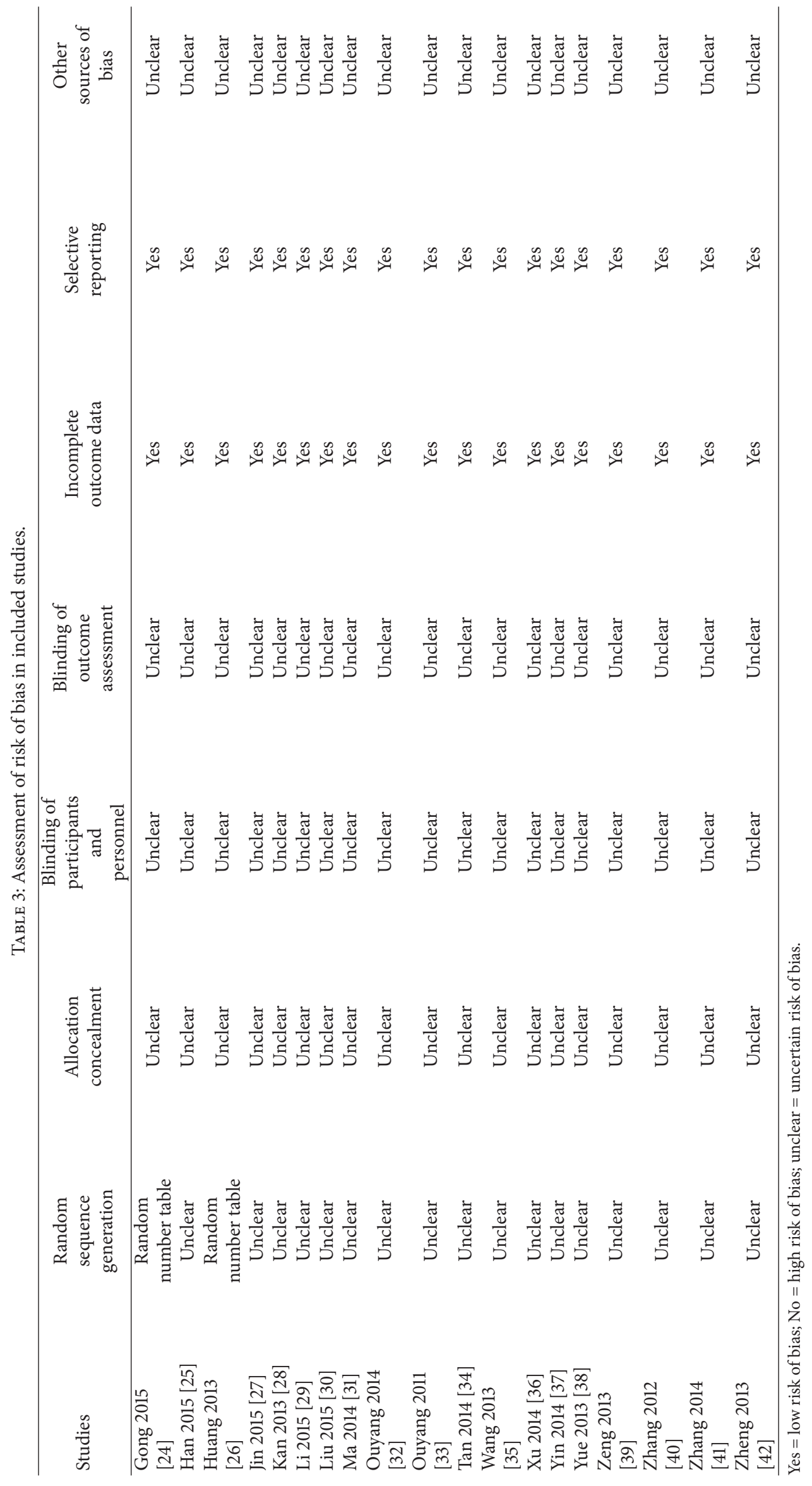




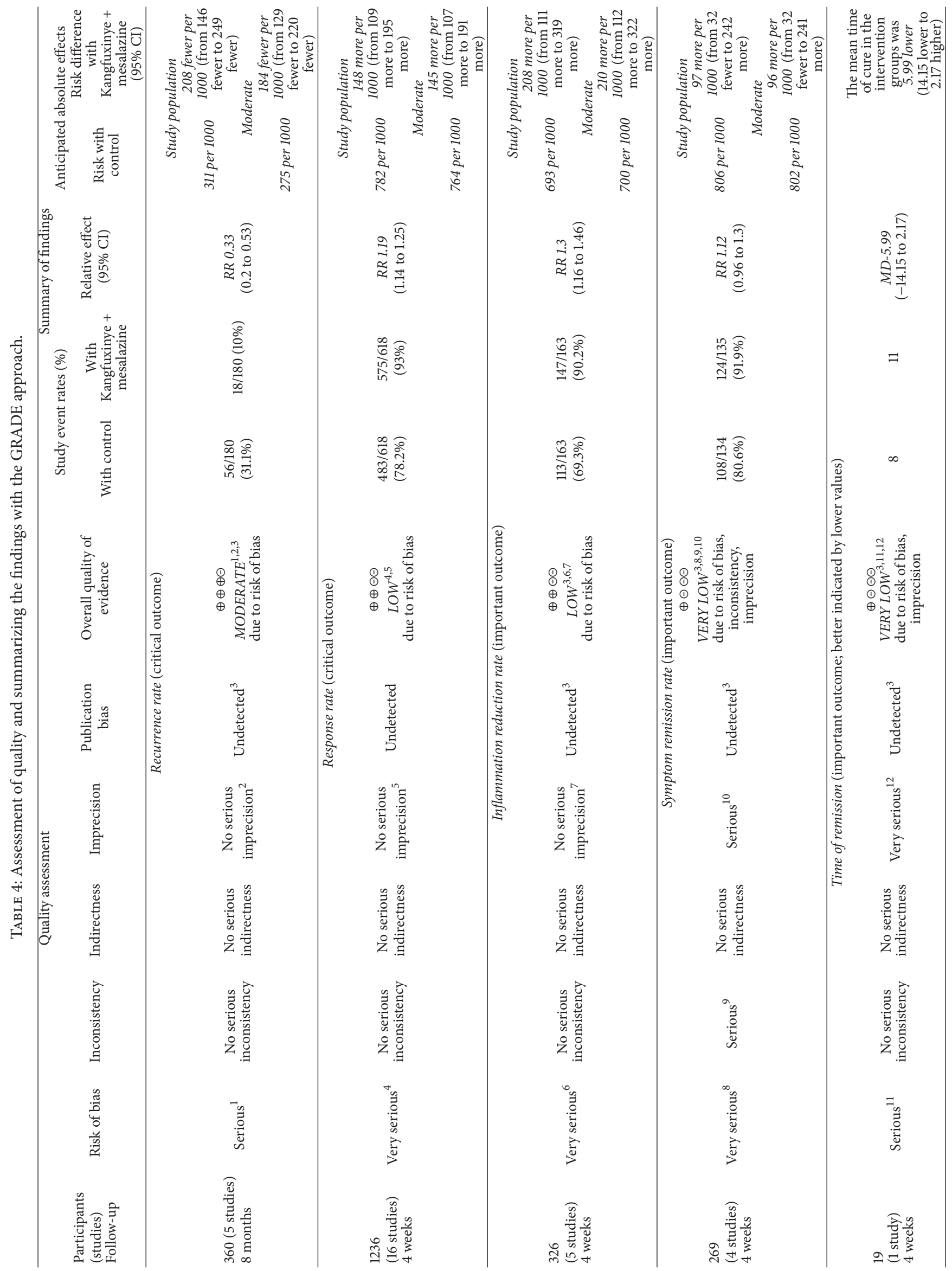




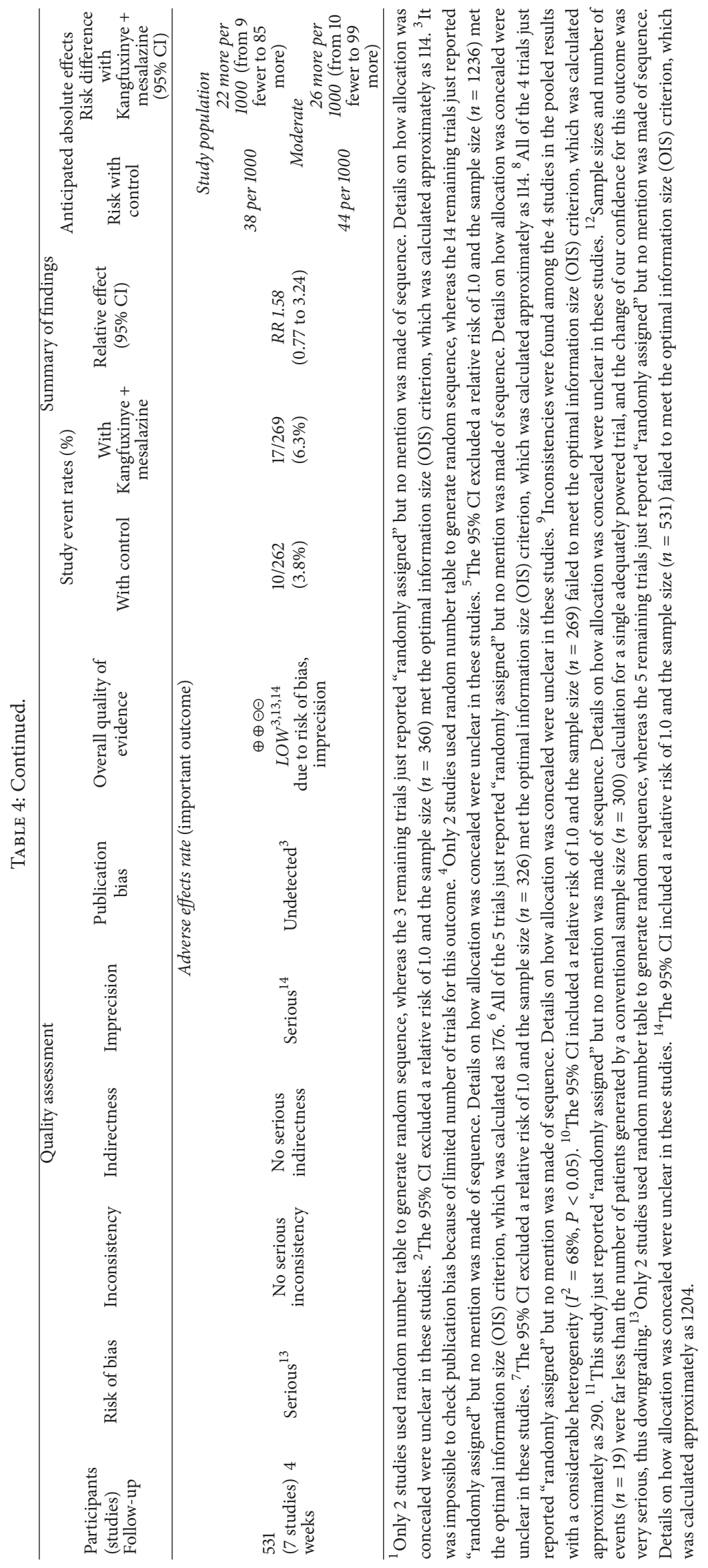




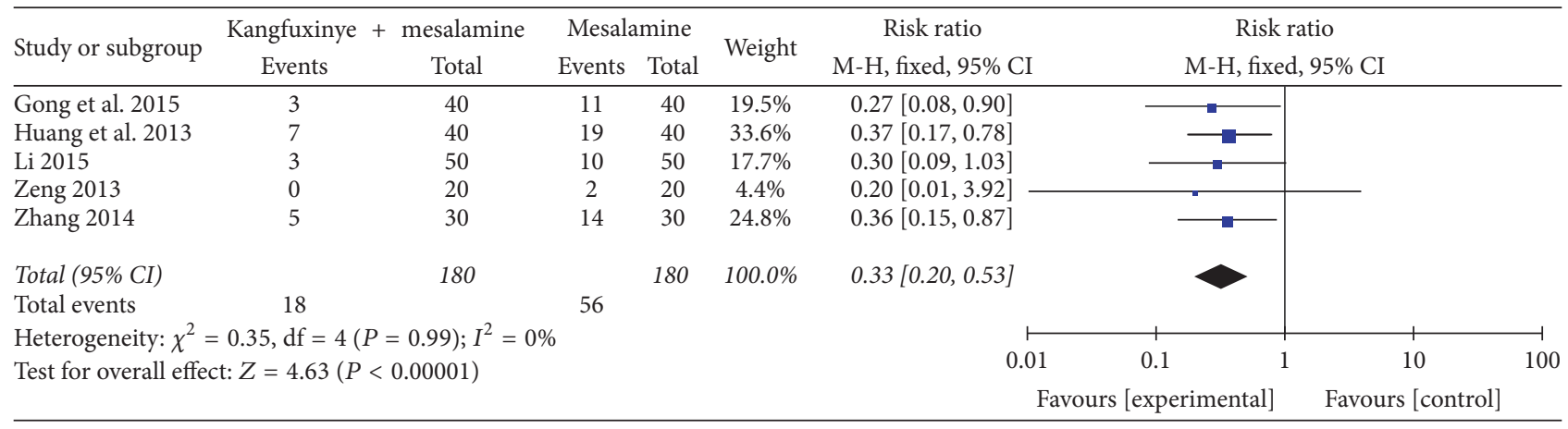

FIGURE 2: Efficacy of Kangfuxinye combined with mesalamine versus mesalamine on recurrence rate.

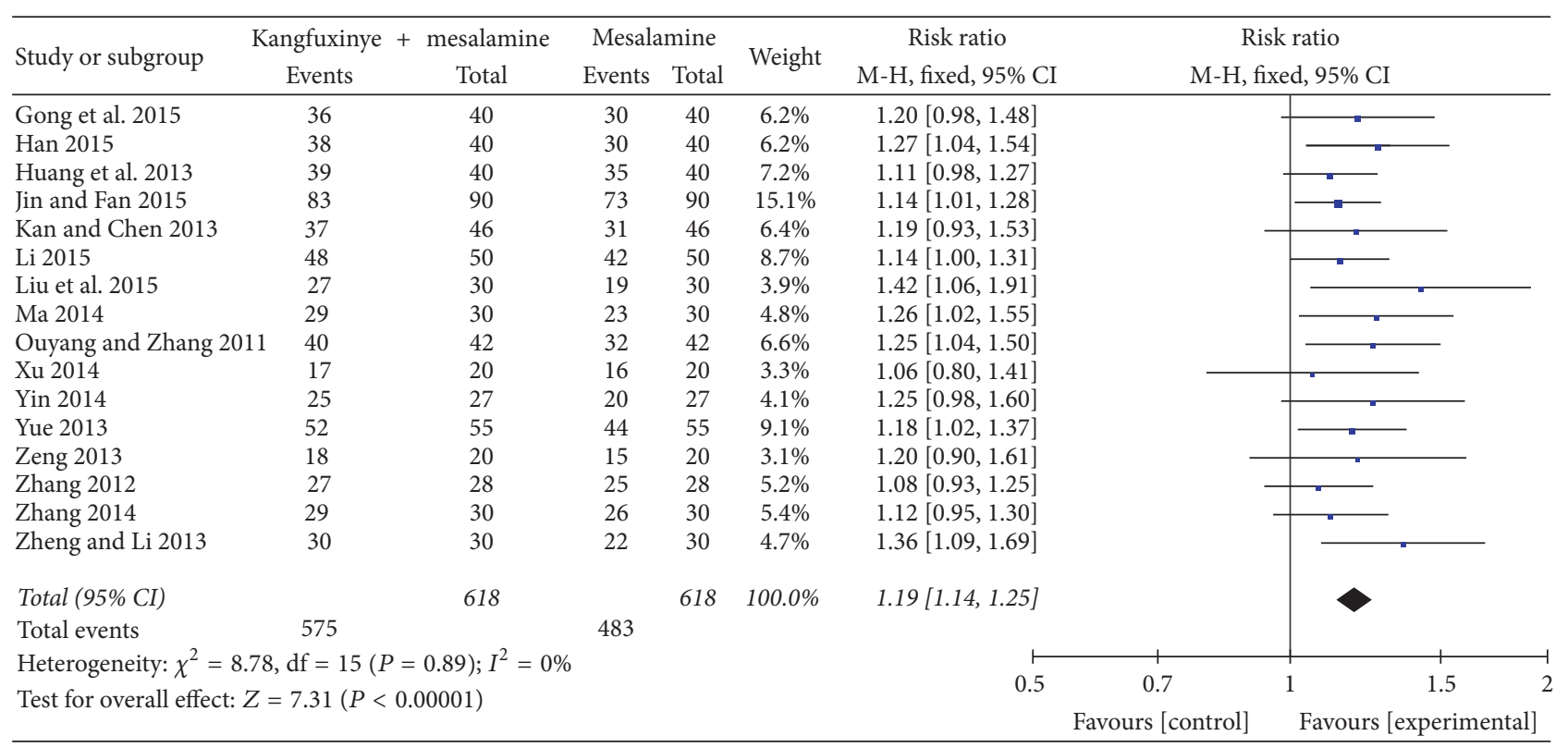

FIgURE 3: Efficacy of Kangfuxinye combined with mesalamine versus mesalamine on response rate.

outcome measure was based on both physician's assessment about the patients' general conditions and the patients' feeling. The meta-analysis indicated that no favourable effects of Kangfuxinye combined with mesalamine compared with mesalamine alone were observed (fixed-effect model, RR = $1.12,95 \% \mathrm{CI}=0.96$ to $1.30, P=0.15)$ with moderate heterogeneity $\left(I^{2}=68 \%, P=0.02\right)$ (Figure 5). A GRADE approach indicated that the quality of evidence supporting this outcome was low due to the risk of bias, imprecision, and inconsistency (Table 4).

3.4.3. Time of Remission. One trial [37] involving 19 participants provided the time of remission, but it failed to present any benefit of Kangfuxinye in terms of shortening time of remission significantly $(\mathrm{MD}=-5.99,95 \% \mathrm{CI}:-14.15,2.17$, $P=0.15$ ) (Figure 6). A GRADE analysis indicated that the quality of evidence supporting this outcome was very low due to high risk of bias and imprecision (Table 4).
3.5. Safety Evaluation. Of the 19 RCTs, 7 trials failed to report anything about adverse events, and the other 12 RCTs [24, 26, $28,29,31-34,38,39,41,42]$ reported adverse events rate. Five trials of those $[33,34,38,39,42]$ reported no adverse events, while at least 1 adverse event was reported in the other 7 trials $[24,26,28,29,31,32,41]$ which included 451 patients that were taken to explore the safety of Kangfuxinye combined with the mesalamine. The meta-analysis showed no difference in Kangfuxinye combined with mesalamine against mesalamine alone (fixed-effect model, $\mathrm{RR}=1.58,95 \% \mathrm{CI}=0.77$ to $3.24, P=0.21)$ without heterogeneity $\left(I^{2}=0 \%, P=0.74\right)$ (Figure 7). A GRADE approach indicated that the quality of evidence supporting this outcome was low due to risk of bias and imprecision (Table 4).

3.6. Publication Bias. Although an asymmetric funnel plot on the response rate was observed, the Egger et al. [48] test failed to identify any publication bias $(P=0.817)$ (Figure 8$)$. 


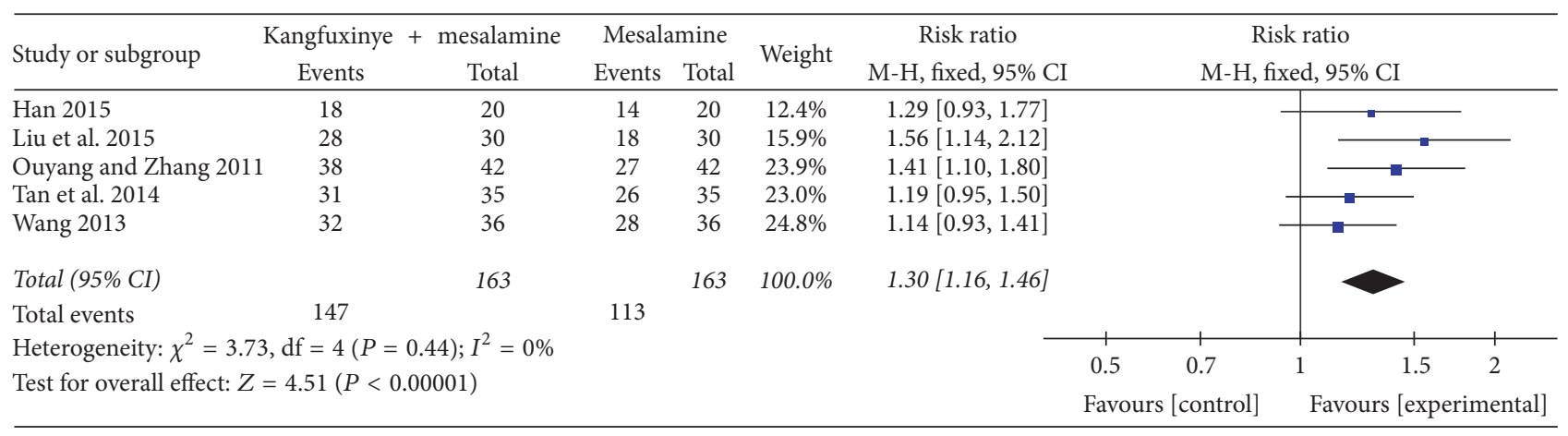

FIGURE 4: Efficacy of Kangfuxinye combined with mesalamine versus mesalamine on inflammation reduction rate.

\begin{tabular}{|c|c|c|c|c|c|c|c|c|c|}
\hline \multirow{2}{*}{ Study or subgroup } & \multicolumn{2}{|c|}{ Kangfuxinye + mesalamine } & \multicolumn{2}{|c|}{ Mesalamine } & \multirow{2}{*}{ Weight } & \multirow{2}{*}{$\begin{array}{l}\text { Risk ratio } \\
\text { M-H, random, } 95 \% \mathrm{CI}\end{array}$} & \multirow{2}{*}{\multicolumn{2}{|c|}{$\begin{array}{c}\text { Risk ratio } \\
\text { M-H, random, 95\% CI }\end{array}$}} & \\
\hline & Events & Total & Events & Total & & & & & \\
\hline Liu et al. 2015 & 27 & 30 & 19 & 30 & $16.0 \%$ & $1.42[1.06,1.91]$ & & & \\
\hline Ouyang and Ran 2014 & 34 & 34 & 32 & 33 & $35.8 \%$ & $1.03[0.95,1.12]$ & & - & \\
\hline Tan et al. 2014 & 30 & 35 & 25 & 35 & $19.5 \%$ & $1.20[0.94,1.54]$ & & & \\
\hline Wang 2013 & 33 & 36 & 32 & 36 & $28.7 \%$ & $1.03[0.89,1.20]$ & & $=$ & \\
\hline \multirow{2}{*}{$\begin{array}{l}\text { Total }(95 \% \text { CI }) \\
\text { Total events }\end{array}$} & & 135 & & 134 & $100.0 \%$ & $1.12[0.96,1.30]$ & & & \\
\hline & 124 & & 108 & & & & & & \\
\hline \multirow{2}{*}{\multicolumn{5}{|c|}{$\begin{array}{l}\text { Heterogeneity: } \tau^{2}=0.02 ; \chi^{2}=9.41, \mathrm{df}=3(P=0.02) ; I^{2}=68 \% \\
\text { Test for overall effect: } Z=1.42(P=0.15)\end{array}$}} & & 0.5 & 0.7 & 1 & 1.5 \\
\hline & & & & & & Fav & Irs [control] & Favours [ex] & sperimental] \\
\hline
\end{tabular}

Figure 5: Efficacy of Kangfuxinye combined with mesalamine versus mesalamine on symptom remission rate.

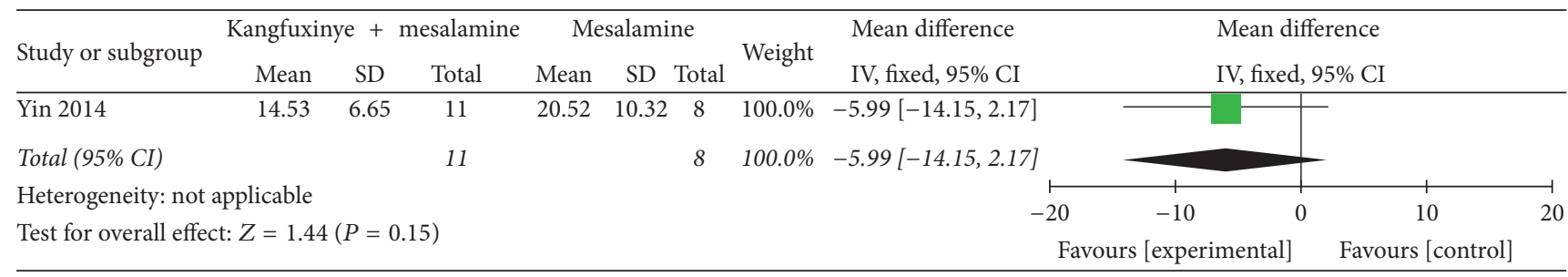

FIGURE 6: Efficacy of Kangfuxinye combined with mesalamine versus mesalamine on time of remission.

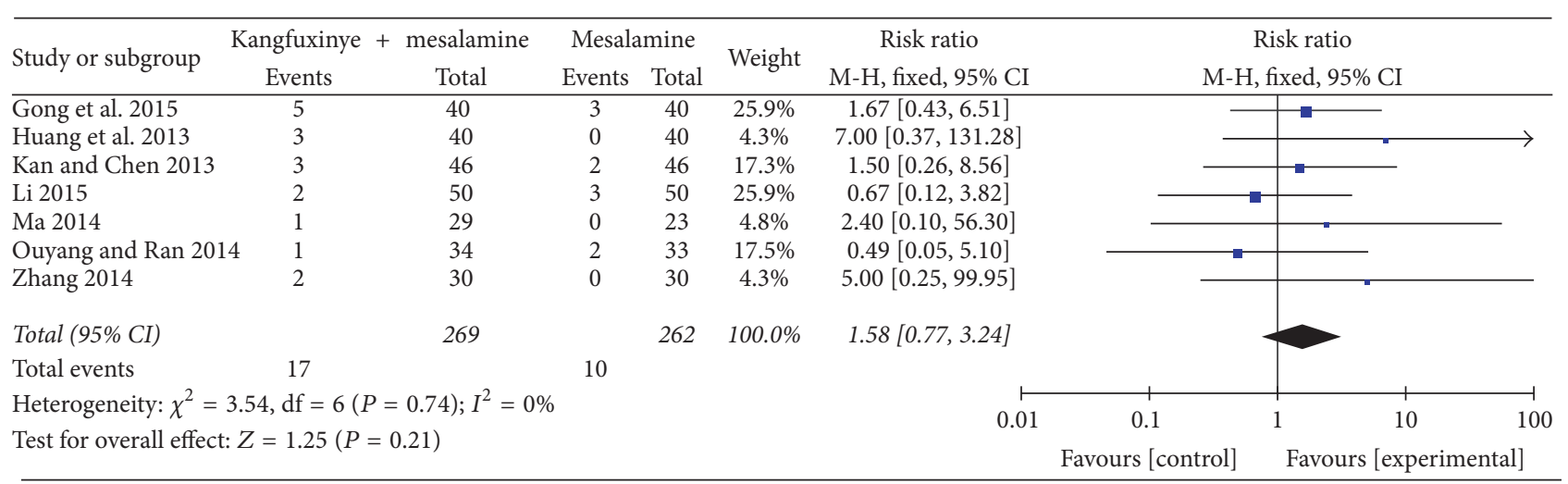

FIgURE 7: Efficacy of Kangfuxinye combined with mesalamine versus mesalamine on adverse events rates. 


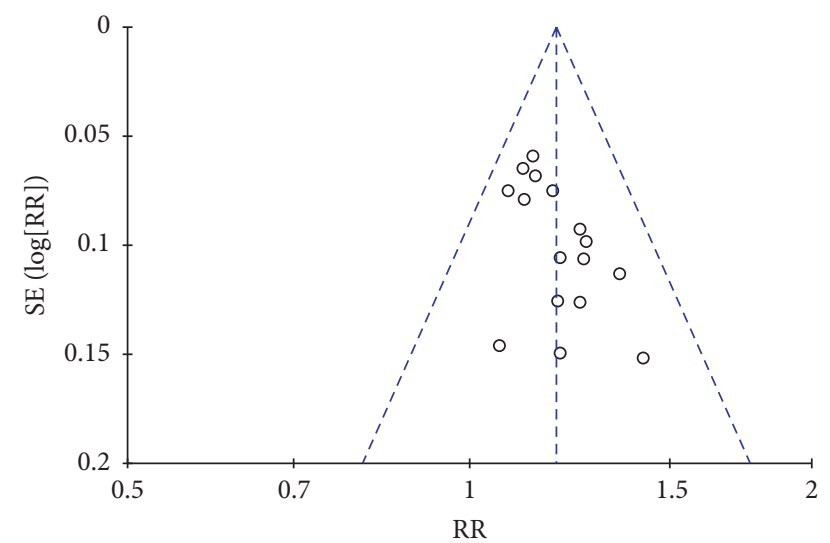

FIGURE 8: Funnel plot analysis on response rate of the 16 trials comparing Kangfuxinye combined with mesalamine versus mesalamine.

\section{Discussion}

19 RCTs involving 1685 patients were identified for this study. The results in our study showed that, compared to mesalamine alone, Kangfuxinye enema combined with mesalamine appeared to be more effective either in reducing recurrence rate or in improving response rate and the inflammation reduction rate. With regard to the symptom remission rate, time of remission, and adverse events rate, no significant benefits were observed. A GRADE approach indicated that most of evidences were rated as moderate, low, or very low quality. Compared with the outcome measured in previous systematic reviews, our review rated rank of relative outcomes according to clinical importance. What is more, the recurrence rate ranked as first of those outcomes due to high relapse rate of UC, and the following in descending order was response rate, inflammation reduction rate, symptom remission rate, and time of remission accordingly in the review.

Furthermore, the quality of evidence on the 6 preset outcomes was rated with the GRADE approach. The evidence of each outcome was downgraded one or two levels due to high risk of bias (poor reporting about randomization and allocation concealment), inconsistency, and imprecision. Five of the 6 outcomes were not downgraded in terms of inconsistency and the remission rate of symptoms was downgraded, which may be explained by heterogeneous patients' characteristics, disease cognition, and susceptibility to adverse events. Regarding imprecision, the OIS referred to the number of participants estimated by a sample size calculation for a single adequately powered trial [50]. If the total number of participants of a meta-analysis is lower than the OIS criterion, the quality of evidence should be downgraded because of imprecision [51]. In this study, the 95\% CIs of the outcomes of adverse effects rate and symptom remission rate included a relative risk of 1.0 [51]; meanwhile the total number of participants for both outcomes $(n=$ 561 and 269, resp.) of the meta-analysis did not exceed conventional sample size $(n=1204$ and 290) calculation for a single adequately powered trial; therefore it was more likely to support downgrading the evidence quality due to imprecision. In addition, as the sample sizes $(n=19)$ of the time of remission were far less than the OIS ( $n=$ 300 ), our confidence in this outcome downgraded two levels. Because there were no significant differences either in baseline characteristics or in the outcomes measured in the included studies, the indirectness was considered as not serious; consequently none of these outcomes was downgraded. Potential publication bias was detected concerning the outcome of inflammation reduction rate through visual inspection. Therefore, the quality of evidence on this outcome was downgraded.

Overall, the quality of evidence with respect to the 6 critical or important outcomes was graded from moderate to very low, and limited data and insufficient follow-up time of long-term effects were more likely to warrant a weak recommendation of Kangfuxinye combined with mesalamine for treating UC patients. Kangfuxinye is crudely extracted by ethanol from dried P. americana whole body and has been approved by the China Food and Drug Administration (CFDA) (Z51021834). The main chemical compositions of Kangfuxinye are amino acids, small molecular peptides, and nucleotides. The present study indicated that $P$. americana extract can increase the levels of prostaglandin E2 (PGE2) [52]. And PGE2 can inhibit acid secretion and increase mucosal blood flow, both of which contribute to the repair of gastrointestinal mucosa [53]. Moreover, it also inhibits the release of inflammatory mediators in the gastric mucosa and inhibits neutrophils, monocytes, and macrophages at inflammatory sites [54]. Therefore, P. americana has a good effect on the gastrointestinal mucosal repairing and anti-inflammatory. A recent study [55] showed that the abstracts enema could accelerate the healing process in dinitrochlorobenzene (DNCB) and acetic acid- (AA-) induced ulcerative colitis rat, whose symptoms and histological features were similar to those of human UC. Moreover, the mechanism was also confirmed that the abstract of $P$. americana was able to encourage fibroblasts proliferation and collagen synthesis in in vitro fibroblast cell model, NIH 3T3 [55]. And a multitude of clinical researches have reported the positive effect of Kangfuxinye. One previous systematic review [21] concerning the clinical application of Kangfuxinye combined with mesalamine in treating UC patients has found that Kangfuxinye could significantly improve the response rate of the UC. However, with only 11 studies retrieved from the Chinese databases, only 2 outcomes (overall response rate and inflammation reduction rate) were taken to perform the pooled analysis, and the adverse events were not pooled due to unavailable data. Moreover, some of the included studies mixed with other interventions in the combination of mesalamine and Kangfuxinye enema.

In our study, a comprehensive literature search was conducted in 7 electronic databases, and, gray literature databases and references lists were taken to identify relevant studies. We also developed explicit eligibility criteria using PICOS (Participants, Intervention, Comparison, Outcome, Study design) format. Only those that compared Kangfuxinye enema combined with mesalamine against mesalamine alone were included. In addition, we graded 6 critical or important 
outcomes according to their clinical importance to grade the quality of evidence by GRADE approach and the recurrence rate was taken as the most critical outcome used to explore the long-term effect of Kangfuxinye enema. Furthermore, we explored the safety of Kangfuxinye enema in terms of adverse events rate. By the way, as we searched relevant databases from the inception through March 31, 2016, the conclusion in our review may be recognized more and up to date, comprehensive, and robust. To the best of our knowledge, this is the first systematic review to grade the quality of evidence and then to generate recommendation regarding the use of Kangfuxinye in UC patients. Currently, rating an overall body of evidence by the GRADE approach is becoming an important and recommended explicit step in evidence synthesis initiatives [56]. With this approach, the details of potential limitations, including risk of bias, result inconsistency, indirectness imprecision, and publication bias are scrutinized for every outcome. And the approach provides us with a structured and transparent way to use this evidence for making a recommendation or decision, particularly for the low or unclear quality of evidence [56]. Therefore, it becomes one of the strengths in our study.

Nevertheless, several limitations should be specially addressed before the acceptance of the findings. Firstly, selection bias may occur in the methodological designs of included studies due to the inadequate reporting, although the review processes were appraised rigorously by 2 experienced and independent authors. Secondly, only two trials $[24,26]$ using a random method divided the groups, and the remaining 17 trials [25, 27-42] reported "randomly allocating" participants but the method of randomization was not described. Thirdly, none of the included trials reported allocation concealment, and whether a blinding method was used or not within 19 trials remains unclear, leading to the increase in risk of selection or performance bias. Last but not least, all included studies were conducted in China and were published in Chinese journals. Although the funnel plot and Egger's regression test failed to detect any publication biases, we could not rule out publication bias absolutely. As studies with statistically significant results are more likely to be published compared to those with null results [57], which seems more common in studies reported in Chinese and other Asian language $[58,59]$, the pooled RR reported in this study may be exaggerated compared to the true value. It is an important threat to the validity of systematic reviews and is difficult to combat except through the registration of all RCTs. In addition, in most studies, the effect of Kangfuxinye enema would be reduced without full contact with the ulcer on account of the fact that enema position of the patients did not vary according to the ulcer locations in the colon. We also noted that the participants of all the trials were all Chinese and whether it is still effective and could be applied to patients outside of China still needs to be further investigated.

\section{Conclusions}

Kangfuxinye enema addition to mesalamine may be effective and safe for UC patients. As the GRADE approach indicated very low to moderate quality of the evidence and lack of information about patients' preference, we suggest a weak recommendation for Kangfuxinye. Considering that all identified studies were of low quality and all were carried out in China, further rigorously designed and large-scale RCTs outside of China are warranted to improve the generalizability and applicability of this study results. And further GRADE approaches are also needed for grading quality of evidence regarding Kangfuxinye in combination with other additional or alternative medicine for UC patients.

\section{Conflicts of Interest}

The authors declare that they have no conflicts of interest.

\section{Authors' Contributions}

Guarantor of the article is De-ying Kang. De-ying Kang conceived and designed the review. Peng-wei Ren, Wenjie Yang, Jing-yan Shan, and Dan-dan Wang conducted literature searches, selected studies, assessed risk of bias, and extracted data. Peng-wei Ren, Wen-jie Yang, and Dan-dan Wang carried out analysis, applied GRADE, and interpreted results. Peng-wei Ren, De-ying Kang, and Qi Hong drafted the manuscript. All authors approved the final version of the manuscript.

\section{Acknowledgments}

The study was supported by Nonprofit Research Project from State Administration of Traditional Chinese Medicine of China, Grant no. 201207005.

\section{References}

[1] A. C. Ford, P. Moayyedi, S. B. Hanauer, and J. B. Kirsner, "Ulcerative colitis," BMJ, vol. 346, article f432, 2013.

[2] "Ulcerative Colitis," NIDDK, September 2014, https://www .niddk.nih.gov.

[3] S. Danese and C. Fiocchi, "Ulcerative colitis," The New England Journal of Medicine, vol. 365, no. 18, pp. 1713-1725, 2011.

[4] A. Ponder and M. D. Long, "A clinical review of recent findings in the epidemiology of inflammatory bowel disease," Clinical Epidemiology, vol. 5, no. 1, pp. 237-247, 2013.

[5] N. A. Molodecky, I. S. Soon, D. M. Rabi et al., "Increasing incidence and prevalence of the inflammatory bowel diseases with time, based on systematic review," Gastroenterology, vol. 142, no. 1, pp. 46-54, e30, 2012.

[6] D. C. Baumgart and W. J. Sandborn, "Inflammatory bowel disease: clinical aspects and established and evolving therapies," The Lancet, vol. 369, no. 9573, pp. 1641-1657, 2007.

[7] C. B. Appleyard, G. Hernández, and C. F. Ríos-Bedoya, "Basic epidemiology of inflammatory bowel disease in Puerto Rico," Inflammatory Bowel Diseases, vol. 10, no. 2, pp. 106-111, 2004.

[8] S. V. Kane, "Systematic review: adherence issues in the treatment of ulcerative colitis," Alimentary Pharmacology and Therapeutics, vol. 23, no. 5, pp. 577-585, 2006.

[9] Z. Zhang and H. Kennedy, "Ulcerative colitis: Current medical therapy and strategies for improving medication adherence," 
European Journal of Gastroenterology and Hepatology, vol. 21, no. 1, pp. 1-8, 2009.

[10] T. M. Letter, Treatment Guidelines, vol. 7 of Drugs for Inflammatory Bowel Disease, The Medical Letter, New York, NY, USA, 2009.

[11] J. Lachaine, L. Yen, C. Beauchemin, and P. Hodgkins, "Medication adherence and persistence in the treatment of Canadian ulcerative colitis patients: analyses with the RAMQ database," BMC Gastroenterology, vol. 13, no. 1, article 23, 2013.

[12] S. C. Truelove and L. J. Witts, "Cortisone in ulcerative colitis; final report on a therapeutic trial," British Medical Journal, vol. 2, no. 4947, pp. 1041-1048, 1955.

[13] Y. Leung, R. Panaccione, B. Hemmelgarn, and J. Jones, "Exposing the weaknesses: a systematic review of azathioprine efficacy in ulcerative colitis," Digestive Diseases and Sciences, vol. 53, no. 6, pp. 1455-1461, 2008.

[14] P. Rutgeerts, W. J. Sandborn, B. G. Feagan et al., "Infliximab for induction and maintenance therapy for ulcerative colitis," The New England Journal of Medicine, vol. 353, no. 23, pp. 24622476, 2005.

[15] L. Langmead and D. S. Rampton, "Review article: complementary and alternative therapies for inflammatory bowel disease," Alimentary Pharmacology and Therapeutics, vol. 23, no. 3, pp. 341-349, 2006.

[16] J. Langhorst, H. Wulfert, R. Lauche et al., "Systematic review of complementary and alternative medicine treatments in inflammatory bowel diseases," Journal of Crohn's \& Colitis, vol. 9, no. 1, pp. 86-106, 2015.

[17] M. Bensoussan, N. Jovenin, B. Garcia et al., "Complementary and alternative medicine use by patients with inflammatory bowel disease: results from a postal survey," Gastroenterologie Clinique et Biologique, vol. 30, no. 1, pp. 14-23, 2006.

[18] X. Xiao, C. Luo, S. Wang et al., "Experimental study on the analgesic and anti-inflammation of periplanetaamericana extract," in The Insects Academic Conference the Fourth National Insects Resource Conference of the Three Provinces in central China (including Hunan, Hubei and Henan), pp. 238-241, Shaoshan Hunan, China, 2005.

[19] H. Chen, X. Lian, L. Jiang, and M. Zhong, "Clinical effect of Kangfuxinliqud in treatment of recurrent aphthous ulcer," China Practical Medicine, vol. 3, pp. 24-25, 2011.

[20] X. H. Liu, A. Bravo-Cuellar, I. Florentin, S. Orbach-Arbouys, G. Mathe, and J. Breard, "In vivo immunopharmacological properties of the traditional chinese medicine Kang Fu-Xin (KFX)," International Journal of Immunotherapy, vol. 5, no. 1, pp. 25-33, 1989.

[21] B. Niu, S. Ye, B. Chen, M. Zhang, D. He, and X. Wang, "Mesalamine combined with Kangfuxin liquid in the treatment of ulcerative colitis: a Meta-analysis," Chinese Traditional Patent Medicine, no. 11, pp. 2275-2279, 2014.

[22] H. J. Schünemann, J. Brożek, G. H. Guyatt et al., GRADE Handbook for Grading Quality of Evidence and Strength of Recommendations, The GRADE Working Group, 2016, http://www.guidelinedevelopment.org/handbook.

[23] G. Guyatt, A. D. Oxman, E. A. Akl et al., "GRADE guidelines: 1. Introduction-GRADE evidence profiles and summary of findings table," Journal of Clinical Epidemiology, vol. 64, no. 4, pp. 383-394, 2011.

[24] J. Gong, C. Yan, and L. Zhang, "Curative efficacy of mesalazine in combination with Kangfuxin solution enema in treatmentof ulcerative colitis and its effects on coagulation parameters," Journal of Armed Police Medicine, no. 5, pp. 440-443, 2015.
[25] J. Han, "A clinical study on treating ulcerative colitis by lavation of Kangfuxin liquid," Journal of Medical Informatics, vol. 28, no. 8, article 236, 2015.

[26] D. Huang, C. Lu, and Z. He, "The combination treatment of mesalamine and Kangfuxinye for the elders ulcerative colitis and its influence on inflammatory cytokines coagulation factors," Chinese Journal of Gerontology, no. 15, pp. 3769-3770, 2013.

[27] J. Jin and Y. Fan, "Clinical research on ulcerative colitis treated with enema of KangfuXinye combined with mesalamine granules," Henan Traditional Chinese Medicine, no. 11, pp. 2725-2727, 2015.

[28] C. Kan and J. Chen, "Clinical observation of Kangfuxin Liquid combined with gentamicin on treatment of ulcerative colitis," Journal of Modern Drugs and Clinical, no. 2, pp. 217-219, 2013.

[29] C. Li, "Observation on curative effect of ulcerative colitis treated with combination of Chinese and Western drugs," Electronic Journal of Clinical Medicine Literature, no. 27, pp. 5730-5731, 2015.

[30] Y. Liu, M. Chen, Z. Lu, and R. Liu, "Effect observation of Kangfuxin solution for retention enema combined with oral administration of mesalazine in the treatment of ulcerative colitis," Journal of Chinese Contemporary Medicine, vol. 22, no. 2, pp. 85-87, 2015.

[31] J. Ma, "Mesalazinecombinated with Kangfuxin for ulcerative colitis," Chinese Journal of Geriatric Care, vol. 12, no. 1, pp. 3738, 2014.

[32] W. Ouyang and H. Ran, "Mesalazinecombinated with Kangfuxin for ulcerative colitis," Chinese Journal of Integrated Traditional and Western Medicine on Digestion, no. 7, pp. 400-401, 2014.

[33] Y. Ouyang and Y. Zhang, "Kangfuxinye enema combined with mesalamine treated for ulcerative colitis," Jilin Medical Journal, vol. 32, no. 16, pp. 3236-3237, 2011.

[34] Z. Tan, S. Zeng, and D. Wang, "Kangfuxinye enema combined with mesalamine treated for ulcerative colitis," Guide of China Medicine, vol. 12, no. 20, pp. 262-263, 2014.

[35] S. Wang, "Kangfuxinye enema combined with mesalamine treated for ulcerative colitis," China Health Care Nutrition, no. 11, article 463, 2013.

[36] N. Xu, "Kangfuxinye enema combined with mesalamine treated for 40 ulcerative colitis patients," China Health Care Nutrition, vol. 24, no. 7, article 4235, 2014.

[37] C. Yin, "The effect foKangfuxinye enema combined with mesalamine treated for ulcerative colitis," Medical Information, no. 28, article 266, 2014.

[38] J. Yue, "Observation the effect foKangfuxinye enema combined with mesalamine treated for ulcerative colitis," Medical Information, no. 16, article 184, 2013.

[39] Q. Zeng, "Observation the effect foKangfuxinye enema combined with mesalamine treated for ulcerative colitis," Chinese and Foreign Medical Research, vol. 11, no. 4, pp. 52-53, 2013.

[40] C. Zhang, "Observation the effect foKangfuxinye enema treated for ulcerative in short time," Clinical Medical Engineering, vol. 19, no. 3, pp. 404-405, 2012.

[41] L. Zhang, "The effect foKangfuxinye enema combined with mesalamine treated for ulcerative colitis," Journal of Clinical Rational Use of Medicines, vol. 7, no. 30, pp. 27-28, 2014.

[42] P. Zheng and C. Li, "A clinical trial of Kangfuxinye enema combined with mesalamine treated for ulcerative colitis," Medical Information, no. 29, pp. 193-194, 2013. 
[43] J. Higgins and G. Sally, Cochrane Handbook for Systematic Reviews of Interventions Version 5.1.0 (updated March 2011), The Cochrane Collaboration, 2011.

[44] D. Moher, A. Liberati, and J. Tetzlaff, "Preferred reporting items for systematic reviews and meta-analyses: the PRISMA statement," Journal of Clinical Epidemiology, vol. 62, no. 10, pp. 1006-1012, 2009.

[45] G. H. Guyatt, A. D. Oxman, G. E. Vist et al., "GRADE: an emerging consensus on rating quality of evidence and strength of recommendations," British Medical Journal, vol. 336, no. 7650, pp. 924-926, 2008.

[46] G. R. Norman, "Issues in the use of change scores in randomized trials," Journal of Clinical Epidemiology, vol. 42, no. 11, pp. 10971105, 1989.

[47] M. Borenstein, LV. Hedges, J. P. T. Higgins, and H. R. Rothstein, Meta-Regression, John Wiley \& Sons, Ltd, 2009.

[48] M. Egger, G. D. Smith, M. Schneider, and C. Minder, "Bias in meta-analysis detected by a simple, graphical test," British Medical Journal, vol. 315, no. 7109, pp. 629-634, 1997.

[49] J. Brożek, A. Nowak, P. Kunstman et al., "GRADEpro Guideline Development Tool (G2DT),” http://www.guidelinedevelopment.org.

[50] J. M. Pogue and S. Yusuf, "Cumulating evidence from randomized trials: Utilizing sequential monitoring boundaries for cumulative meta-analysis," Controlled Clinical Trials, vol. 18, no. 6, pp. 580-593, 1997.

[51] G. H. Guyatt, A. D. Oxman, R. Kunz et al., "GRADE guidelines: 6. Rating the quality of evidence-imprecision," Journal of Clinical Epidemiology, vol. 64, no. 12, pp. 1283-1293, 2011.

[52] H. W. Zhang, L. Y. Wei, Z. Y. Zhang, S. Z. Liu, and J. Zhang, "Preventive effect of periplaneta americana extract on stress ulcer bleeding in patients with acute lung injury or acute respiratory distress syndrome," Chinese General Practice, vol. 15, pp. 3878-3879, 2012.

[53] Y. Li, H. W. Wu, and X. J. Dong, "Mechanism of kangfuxin solution on gastric ulcer," Chinese Remedies \& Clinics, vol. 8, pp. 495-496, 2008.

[54] X. Q. Xiao, S. P. Wang, C. Luo, S. R. Xu, S. J. Wu, and F. Zhong, "Preliminary study of periplaneta americana extract on gastric ulcer," Journal of Tropical Medicine, vol. 6, pp. 1274-1276, 2006.

[55] N. Li, R. Lu, Y. Yu et al., "Protective effect of Periplanetaamericana extract in ulcerative colitis rats induced by dinitrochlorobenzene and acetic acid," Pharmaceutical Biology, vol. 54, no. 11, pp. 2560-2567, 2016.

[56] P. Alonso-Coello, H. J. Schünemann, J. Moberg et al., "GRADE Evidence to Decision (EtD) frameworks: a systematic and transparent approach to making well informed healthcare choices. 1: introduction," BMJ, vol. 353, article i2016, 2016.

[57] K. Dickersin, S. Chan, T. C. Chalmersx, H. S. Sacks, and H. Smith Jr., "Publication bias and clinical trials," Controlled Clinical Trials, vol. 8, no. 4, pp. 343-353, 1987.

[58] J. Liu, E. Manheimer, Y. Shi, and C. Gluud, "Chinese herbal medicine for severe acute respiratory syndrome: a systematic review and meta-analysis," Journal of Alternative and Complementary Medicine, vol. 10, no. 6, pp. 1041-1051, 2004.

[59] P. Jüni, F. Holenstein, J. Sterne, C. Bartlett, and M. Egger, "Direction and impact of language bias in meta-analyses of controlled trials: empirical study," International Journal of Epidemiology, vol. 31, no. 1, pp. 115-123, 2002. 


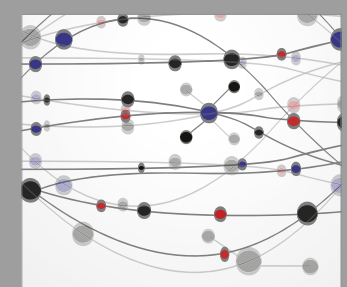

The Scientific World Journal
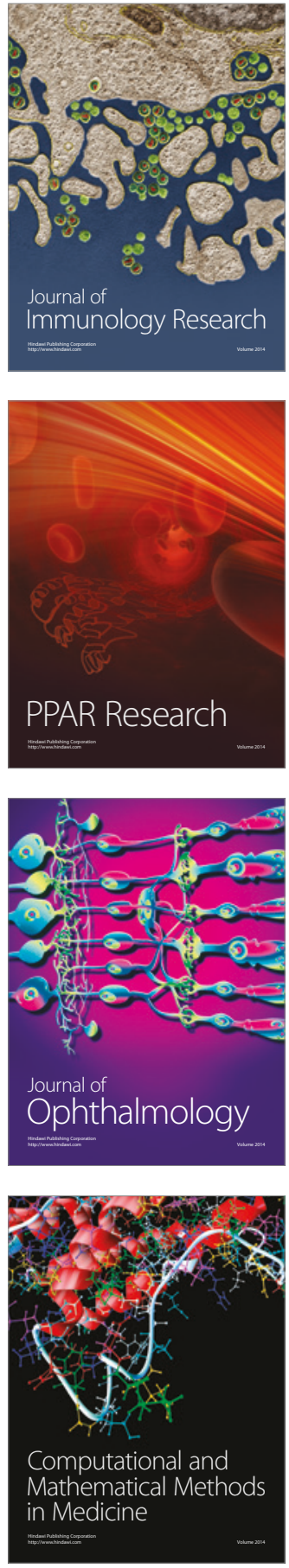

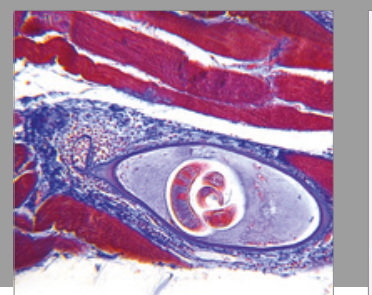

Gastroenterology Research and Practice
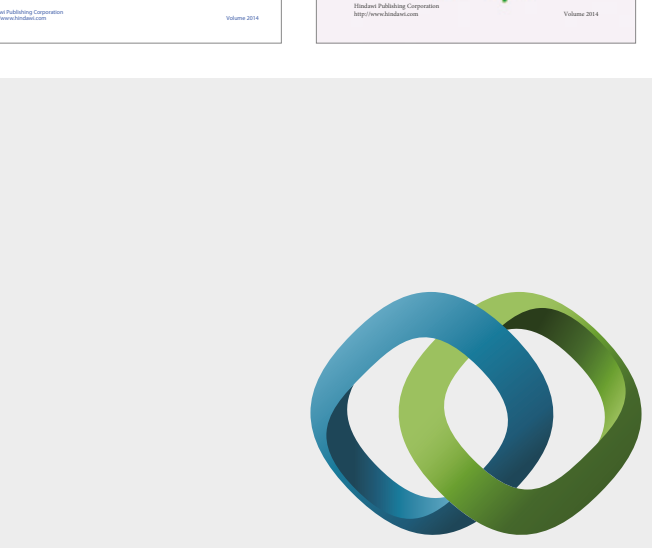

\section{Hindawi}

Submit your manuscripts at

https://www.hindawi.com
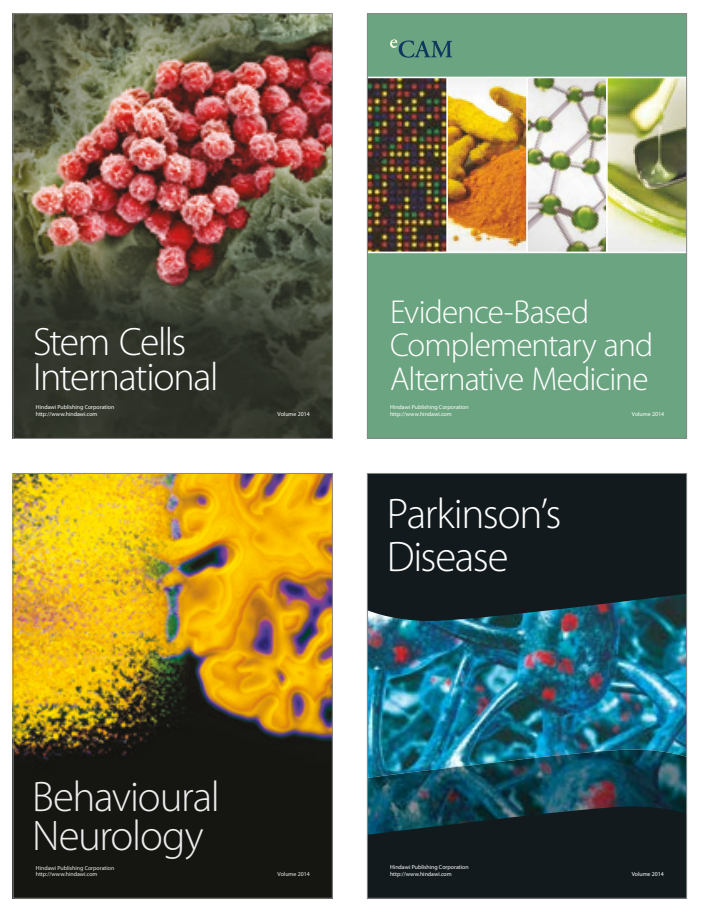
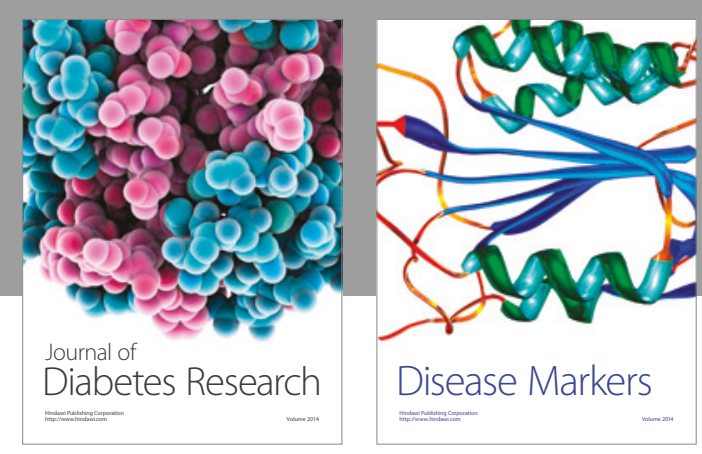

Disease Markers
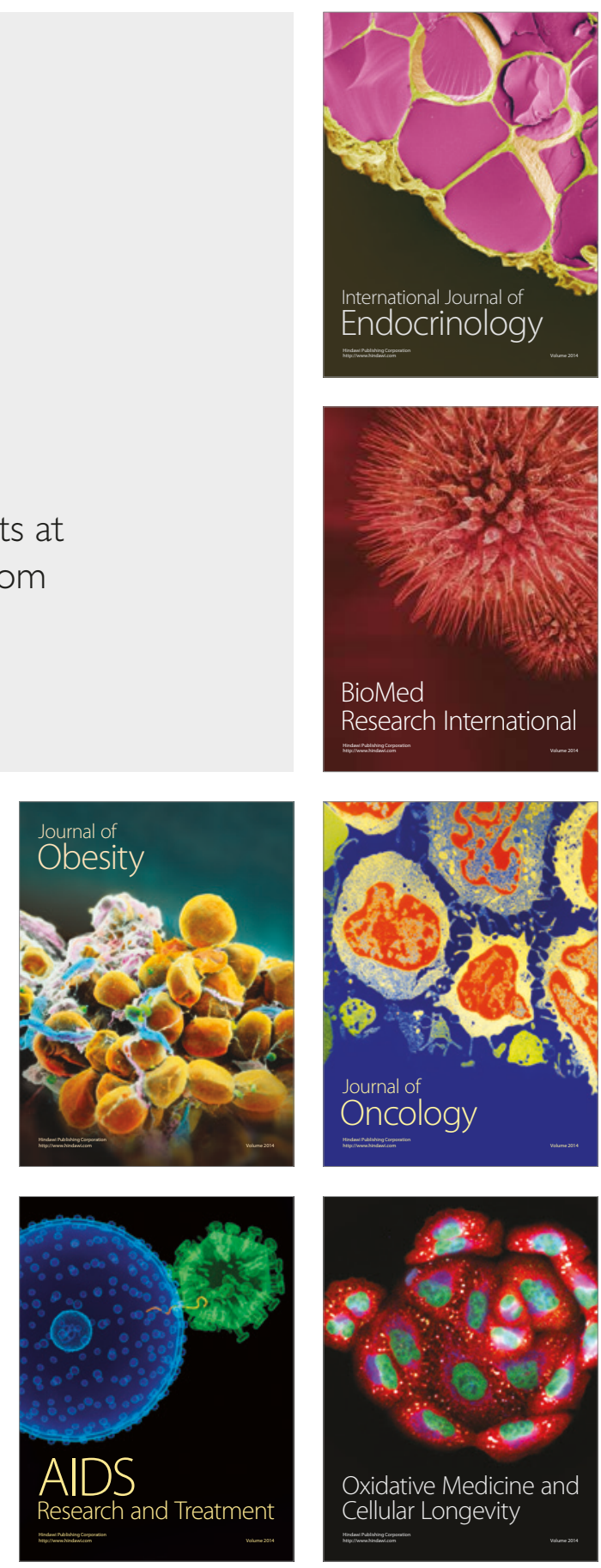